\title{
Aesthetic Delusions: An Investigation into the Role of Rapid Visual Adaptation in Aesthetic Practice
}

\author{
Kate Goldie \\ David Cumming ${ }^{2,3}$ \\ Daria Voropai (iD \\ Afshin Mosahebi ${ }^{4,5}$ \\ Sabrina Guillen Fabi ${ }^{6,7}$ \\ Claus-Christian Carbon (iD) 8,9 \\ 'European Medical Aesthetics, London, \\ UK; ${ }^{2}$ Oxford Al Ltd, Oxford, UK; \\ ${ }^{3}$ Swinburne University of Technology, \\ Melbourne, Australia; ${ }^{4}$ Division of \\ Surgery \& Interventional Science, Faculty \\ of Medical Sciences, University College \\ London, London, UK; ${ }^{5}$ Department of \\ Plastic Surgery, Royal Free Hospital, \\ London, UK; ${ }^{6}$ Cosmetic Laser \\ Dermatology, San Diego, CA, USA; \\ ${ }^{7}$ University of California, San Diego, CA, \\ USA; ${ }^{8}$ Department of General \\ Psychology and Methodology, University \\ of Bamberg, Bamberg, Germany; \\ ${ }^{9}$ Research Group EP/EG (Ergonomics, \\ Psychological / Æsthetics, Gestalt), \\ Bamberg, Germany
}

Background: To date, the process of adaptation in the setting of aesthetic medicine has not been investigated. The combination of complex advanced feedback in the current intense social media milieu, in conjunction with easily accessible and effective aesthetic treatments, has produced pockets of overtreated patients and over-zealous practitioners. We examine whether aesthetic assessments of attractiveness and what appears natural can be distorted by the cognitive process of adaptation.

Methods: Forty-eight female participants were exposed to photographs of female faces in whom lip fullness had been strongly under- or over-exaggerated, while remaining within the bounds of natural appearing lips. Before and after evaluation of the exaggerated images, participants were asked to rate an alternative set of faces in terms of attractiveness (reflecting direct assessment of effective beauty impression) and naturalness (reflecting indirect assessment of beauty norms). The evaluation set consisted of six base faces that had been digitally altered to create a systematically varying 11 step set of lip sizes from extremely thin, to the original version, to very full.

Results: Second-order polynomial fits indicated clear shifts of the subjects' facial aesthetic assessments towards the specific lip fullness of the adaptors. In contrast, such adaptions were not found for ratings of face naturalness. In contrast to research demonstrating mathematical foundations and unchanging rules governing perceptions of beauty, we show that simple viewing of exaggerated feature morphologies can rapidly result in recalibration of a person's assessment of attractiveness.

Conclusion: This paper provides evidence that facial attractiveness is fluid, and that there are psychological mechanisms that cause an aesthetic bias. Over-exposure to exaggerated features can lead to significant changes to a person's ideas of attractiveness.

Keywords: aesthetic assessments, attractiveness, standards, norm, flexibility, adaptation, facial attractiveness

\section{Plain Language Summary}

This study evaluated whether a person's aesthetic appreciation of faces would adapt after exposure to images of strongly modified lips (very full versus very thin). Presentation of strongly manipulated full lip images resulted in participants' highest rating of attractiveness being shifted towards faces with fuller lips, while presentation of thin-lip images shifted attractiveness rating towards faces with thinner lips. These findings demonstrate that an individual's aesthetic preference was affected by viewing extreme image versions after only a short exposure, illustrating how manipulated media can alter norms of attractiveness. 


\section{Introduction}

In the 21st Century electronic culture, the visual diet is a feast of manipulated images, with Instagram filters, Photoshopping, airbrushing Apps, all distorting, reshaping, beautifying, while pushing norms of appearance to extremes. Manipulated images can profoundly affect a person's perception. ${ }^{1,2}$ The underlying cognitive processing is automatic and can lead to lower satisfaction with the self-image, ${ }^{2,3}$ and changes in our visual habits, standards, and altered internalized norms of facial beauty. ${ }^{4}$ Such altered perceptions can result in the adaptation of mental standards and norms towards the observed stimulus images. ${ }^{5}$ Adaptations to visual stimuli have been documented for many aspects of face perception such as facial identification, ${ }^{6}$ the perception of facial dimensions, ie, the "plausibility" of a face, ${ }^{7}$ and also assessments of beauty and attractiveness. ${ }^{5,8}$ These adaptations have been documented to form in seconds to minutes, ${ }^{9,10}$ and persist for days, ${ }^{11}$ and up to 1 week. ${ }^{12}$

Just inspecting certain facial stimuli might, via the socalled "transfer effect", change the perception of other faces for as long as the adaptation lasts. ${ }^{14,15}$ This type of adaptation also appears to be very robust against situational changes, and does not seem to reflect mere episodic artefacts, but changed mental representations. ${ }^{13}$ Fast and flexible adaptation, updating mental representations ${ }^{16}$ with the latest information from personal experience (ie, the psychological environment ${ }^{17}$ ) is a very useful mental ability.

The toxic component of adaptation, however, is that the process can be triggered by irrelevant, unrealistic, and even, absurd visual information such as the perception of strongly modified faces and body images, ${ }^{18}$ unrealistic role models; ${ }^{19}$ and body shapes. ${ }^{20}$ These maladaptive distortions of otherwise useful mental functionalities can lead via a "runaway" process to more and more extreme delusions, a lowering of self-esteem, ${ }^{21}$ and dissatisfaction with body self-image. ${ }^{22}$

The aim of the present study was to investigate the process of adaptation by exposing subjects to facial images with extremely modified lips. Adaptation was then assessed by evaluating the impact on the subjects' personal beauty preferences as indicated by the subjects' assessments of facial attractiveness and facial naturalness.

\section{Method}

\section{Rationale}

The aim of the present study was to explore mechanisms of adaptation in perceptions of lip fullness and associated facial aesthetic appreciation. We considered the impact of these changeable perceptions on assessments in medical aesthetics. Does a person's aesthetic appreciation of faces adapt, within a short session of exposure, to strongly modified lips - in this case, fuller vs thinner lips? To test this, we employed two measures of aesthetic appreciation: 1) Face attractiveness, which reflects a direct assessment of beauty impressions and 2) naturalness, which reflects an indirect assessment of beauty norms and relates to more sustainable beauty standards. We considered whether adaptation impacts only more short-term, effective beauty impressions or also long term, beauty standards.

\section{Participants}

Female volunteers with normal or corrected-to-normal vision (Snellen test) and normal color vision (short version of the Ishihara test) were recruited and randomly assigned to the two experimental conditions: Adapt_FullLips or Adapt_ThinLips. This study recruited female participants only as previous studies have indicated that observers are more sensitive to facial changes affecting their own gender, ${ }^{23}$ here of uniform lip manipulations of female faces. Participants were naïve to the purpose of the study and were tested individually. Informed written consent was obtained from all participants. All procedures were in accordance with the national ethical standards on human experimentation and with the Declaration of Helsinki of 1975, as revised in 2008. Additionally, the study was approved by the local ethics committee of the University of Bamberg on 22 August 2019 under dossier number \#2019-08/22.

\section{Stimuli}

We used twelve frontal, high-quality photographic portraits as base faces. These were natural facial images of twelve different women who had not undergone any kind of facial plastic surgery. The original photographs were initially graphically optimized by removing very large paraphernalia (eg, long earrings) and any kind of lettering or advertisements on clothing to minimize any distraction by such visual cues. In a subsequent step, the images were cropped to a height of 660 pixels ( 72 pixels per inch) with the face centered in the frame and occupying the majority of the space. The width of the images was between 495 and 573 pixels, depending on the original width of the respective face. Using these standardized base faces, additional variants were created that held constant all physical parameters of the images with the exception of lip fullness. 
Lip fullness was manipulated using the Face Liquify tool of Adobe Photoshop. Five variants of each base face were produced with increasingly thinner lips than the base, and five variants with increasingly thicker lips than the base. The Face Liquify tool offers ranges from $0 \%$ (normal) to $100 \%$ (maximum change) in change of lip fullness. We decided to create both thinner and fuller lip sets in steps of $20 \%$ to reach equidistant differences among subsequent variants. Variants were termed M100 (minus 100, ie, thinnest possible lips), M80 (minus 80 ), M60, M40, M20, P20 (plus 20, ie, $+20 \%$ fuller lips than the original), P40 (plus 40), P60, P80, and P100 (plus 100 , ie, fullest possible lips) (Figure 1). In total, the set comprised 12 [base faces] $\times(1$ [original] +5 [thinner variants] $+5[$ fuller variants $])=132$ face stimuli. The total set was divided in two same sized groups that comprised all variants of six base faces each. This was performed to obtain an adaptor set and an evaluation set of stimuli. For every second participant, we changed the assignment of adaptor vs evaluation set. For the adaptor set, we only employed the maximum variants of the respective six base faces, ie, the $\mathrm{P} 100$ variants for the Adap_FullLips adaptation condition and the M100 variants for the Adapt_ThinLips adaptation condition. For the evaluation set, we took the entire range of eleven variants of the respective six base faces.

\section{Apparatus}

For the experimental testing ie, the adaptation and evaluation phases, a standard PC was used with a 22-inch colour- calibrated monitor running at a screen resolution of $1024 \times 768$ pixels. Key presses were registered by an ordinary keyboard. A set of questionnaires to gain deeper knowledge of the participants was used to exclude any extreme psychological dispositions, for example, people who seek sensation by extreme body modifications or by highly extravert behavior. To screen for sensation-seeking, we developed a test based on the logic of the revised SSS$\mathrm{V}^{24}$ In total, this test consisted of 39 items utilizing a 7-point Likert scale. To test personality traits, we used the short version of the Big Five Inventory (BFI) with 21 items. ${ }^{25}$ Participants' attitudes to plastic surgery were screened through a self-developed questionnaire with 15 items employing a uniform 7-point Likert rating scale from 1 (does not apply at all) to 7 (does apply perfectly). The full questionnaire in German along with an English translation can be found in the Supplementary Table 1.

\section{Procedure}

To investigate the impact of viewing faces with extreme versions of lips on participants' subsequent evaluation of 1) facial attractiveness, and 2) face naturalness, the experiment consisted of test Phase 1 (T1), adaptation phase (Adaptation) and test Phase 2 (T2). In T1 and T2, respectively, participants were exposed to one stimulus after another, all originating from the selected face evaluation set.

Both test phases (T1 and T2) consisted of two blocks, the first addressed face attractiveness and the second

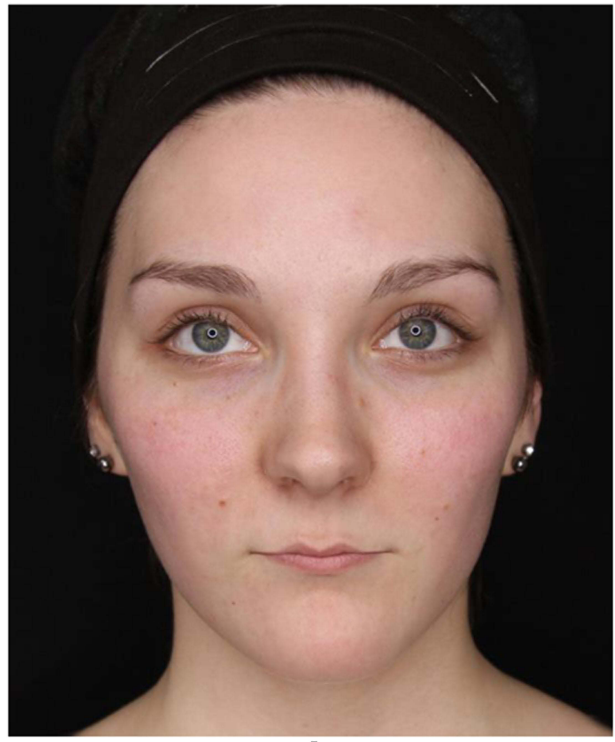

A

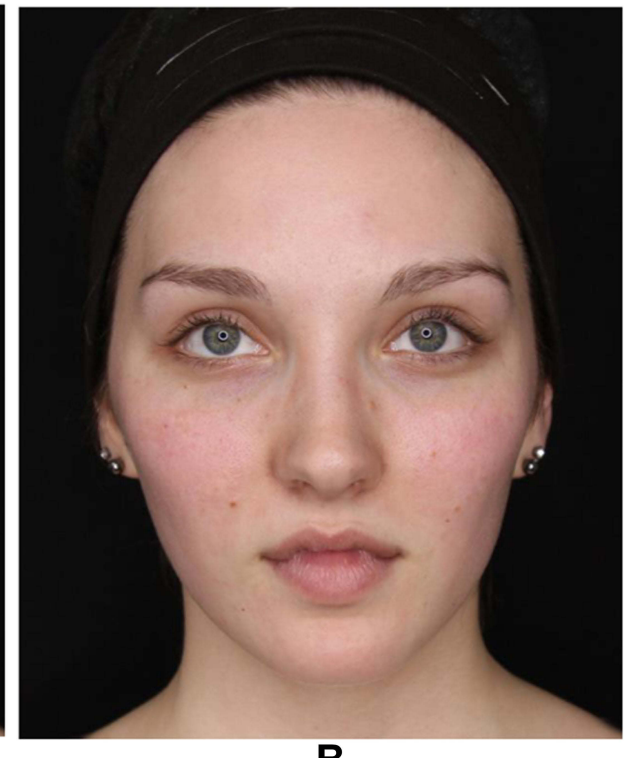

B

Figure I Lip fullness was manipulated using the Face Liquify tool of Adobe Photoshop and ranged from (A) thinnest possible lips variant (minus I00) to (B) fullest possible lips variant (plus 100). 
addressed face naturalness. Facial attractiveness was used as an indicator for individual aesthetic assessment, and face naturalness as an indicator of feasible morphology of the subject's experience. For each block, we randomized the order of presentation. Each stimulus was presented in the center of the screen and participants made their evaluation by pressing a key between 1 and 7 indicating their judgement from very low to very high. T2 was in fact a repetition of $\mathrm{T} 1$ and allowed any changes in the two variables caused by the intermediate adaptation phase to be measured. In the adaptation phase, we presented the selected face adaptation set (either faces with full lipscondition FullLips, or faces with thin lips-condition ThinLips) employing the Repeated Evaluation Technique $\left(\mathrm{RET}^{26}\right)$, a paradigm used to ascertain deep elaboration of target material through a series of evaluations performed in the same sequence. In the present RET, participants were required to evaluate each face regarding five variables in a similar manner. The order of the variables was always the following: 1) skin quality, 2) pigmentation, 3) youthfulness, 4) femaleness, and 5) hair quality. Presentation of the stimuli on-screen was identical to presentation in the test phases. At the end of the experiment, immediately after T2, participants were asked to rate lip fullness for all presented stimuli. They used the same generic 7-point scale as in the preceding phases.

Participants completed the written consent, the BFIK21 , and the psychological screening questionnaires before performing the test phases. After the experiment, participants completed the self-developed questionnaire on attitudes to plastic surgery. The entire procedure took approximately 45-60 min. Participants received course credit for their participation.

\section{Statistical Analysis}

The data were processed by RStudio 1.2.5033 employing $\mathrm{R}$ 3.6.2. The $\mathrm{R}$ package psych was utilized for calculating effect sizes. Analysis of Variance (ANOVA) and linear mixed effects analysis were conducted via package lme4. ${ }^{27}$

All assessment data with response times less than 300 ms and greater than 4 SDs above the individual mean RT level, specifically calculated for each dependent variable and test phase, were identified as outliers and consequently excluded from further data analysis. ${ }^{28}$

In order to check the implementation of the lip manipulations, the native levels of lip fullness offered by the graphics software (from -100 to +100 ) were compared with the participants' assessments of lip fullness at the end of the experiment (assessed on a scale from 1 to 7 ) and Pearson R coefficient was calculated for both experimental conditions.

The mean values of the two dependent variables 1) face attractiveness and 2) face naturalness for both adaptation conditions (Adapt_FullLips and Adapt_ThinLips) were fitted to second-degree polynomial functions with the determination coefficient expressed as Pearson's $R^{2}$.

\section{Results}

A total of 48 female volunteers (mean age $\left[M_{\text {age }}\right]=22.5$ years, $S D=7.2)$ participated in the study: 25 were randomly assigned to Adapt_FullLips $\left(M_{\mathrm{age}}=22.1\right.$ years, $\left.S D=7.0\right)$, and 23 to Adapt_ThinLips $\left(M_{\mathrm{age}}=22.9\right.$ years, $\left.S D=7.4\right)$; there was no significant difference in age between the two sub-samples (unpaired two-sided $t$-test, $p=0.6976$ ).

Pearson $R$ coefficient for both experimental conditions based on mean data indicated close to perfect fit with $R^{2}>$ 0.996 (Figure 2). A nearly perfect linear relationship between physical and psychological lip fullness for our experimentally manipulated material can therefore be assumed.

The mean values of the two dependent variables, face attractiveness and face naturalness, followed a trend that was very close to perfect second-order polynomial functions $\left(R^{2}>0.965\right)$ based on lip fullness as the independent variable (Figure 3 ). This indicates that the more strongly lips were modified (towards very thin as well as towards very full lips), the less attractive and the less natural they were assessed. Furthermore, adaptation had a clear impact on the assessments of attractiveness but not on naturalness. Whereas the naturalness assessments at T2 were in fact nearly in perfect registry with $\mathrm{T} 1$, an upwards shift of the entire assessment level for the attractiveness task through adaptation was noted.

Most importantly, this general upward shift in attractiveness assessments from $\mathrm{T} 1$ to $\mathrm{T} 2$ showed movement in both directions, shifting towards the adaptors in both cases. Participants' highest rating of attractiveness shifted towards faces with fuller lips in the case of the Adapt_FullLips condition, while participants' highest attractiveness assessments were shifted towards faces with thinner lips in the adaptation condition Adapt_ThinLips.

We statistically tested these selective adaptation effects for attractiveness vs naturalness on the basis of the maximal assessments retrieved from the modelled data for each person, adaptation condition and test phase. As models, we used second-order polynomial functions. Maxima 


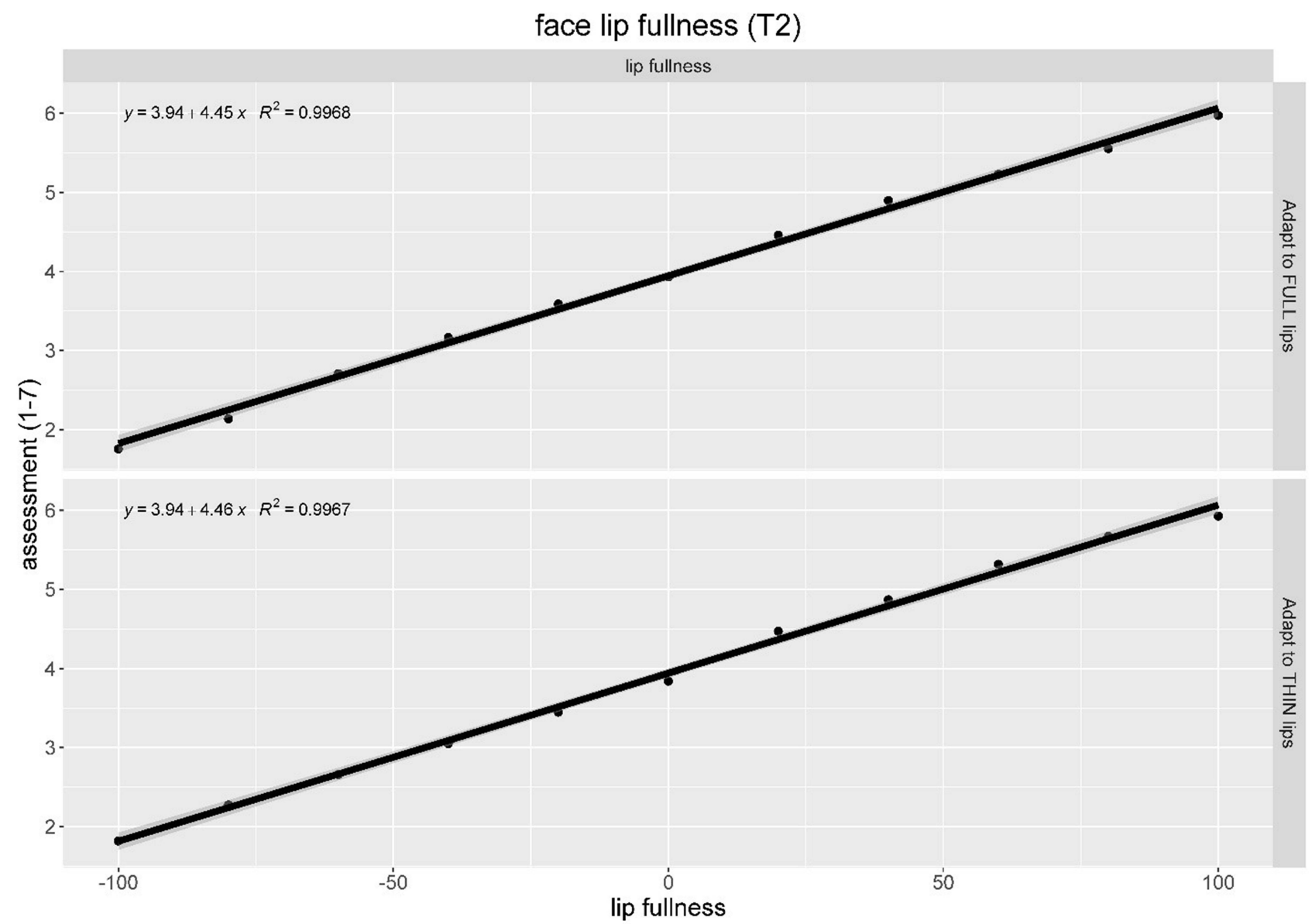

Figure 2 Comparison of presented levels of lip fullness offered by the graphics software ( $x$-axis: from -100 to +100$)$ with the participants' assessments of lip fullness at the end of the experiment ( $y$-axis: from I to 7). Pearson R coefficient for both experimental conditions indicate close to perfect fits. Confidence intervals (Cl-95\%) are additionally given by shadowed confidence bands (note: the band can hardly be perceived as it is in fact very narrow due to the near-to-perfect fit).

were identified by calculating the first derivatives of the respective polynomials. As outlier criteria for the maxima, we used all values outside of a natural range of $[-60,+60]$. Only very few of the curve fittings resulted in outliers as shown by the corresponding effective $n$ of maxima interpretations which could be further processed (Table 1).

We further analysed the maxima by means of two independent mixed design ANOVA employing the lmer routine by $\mathrm{R}$, one for the assessments of attractiveness, and the other for naturalness. We used Adaptation (Adapt_FullLips, Adapt_ThinLips) and Test phase (T1, T2) plus the interaction between both variables as fixed slope effects, and participants were taken as random intercept effects. Degrees of freedom were calculated following the Welch-Satterthwaite equation.

For attractiveness, we obtained significant effect for the large interactive effect between Adaptation and Test phase, $F(1,40.2)=11.0, p=0.0019$, Cohen's $f=0.460$. All other effects were at $p>0.2109$. For naturalness, no effects were found to be significant, $p$ values $>0.3796$. The interactive effect for attractiveness between the factors adaptation and test phase (see Figure 4) was further investigated by subsequent directed paired $t$-tests between $\mathrm{T} 2$ and $\mathrm{T} 1$ for all combinations of the dependent measures and Adaptation (see Table 1). We obtained significant adaptations of attractiveness assessments in both Adapt_ThinLips and Adapt_FullLips conditions. Generally, Adapt_ThinLips scored highest in assessment changes. One can conjecture that the runaway effect is greater with full lips in our society because we do not have a satisfactory way of reducing lip size. In essence, our treatment regimens provide a one-way gate to lip fullness.

To test the contrastive effect of the two different adaptation sets, extremely full lips (Adapt_FullLips) vs extremely thin lips (Adapt_ThinLips), we conducted unpaired $t$-tests between both adaptation conditions fixed at test phase T2. A medium-to-large effect was obtained for attractiveness, Cohen's $d=0.686$, indicating a selective 


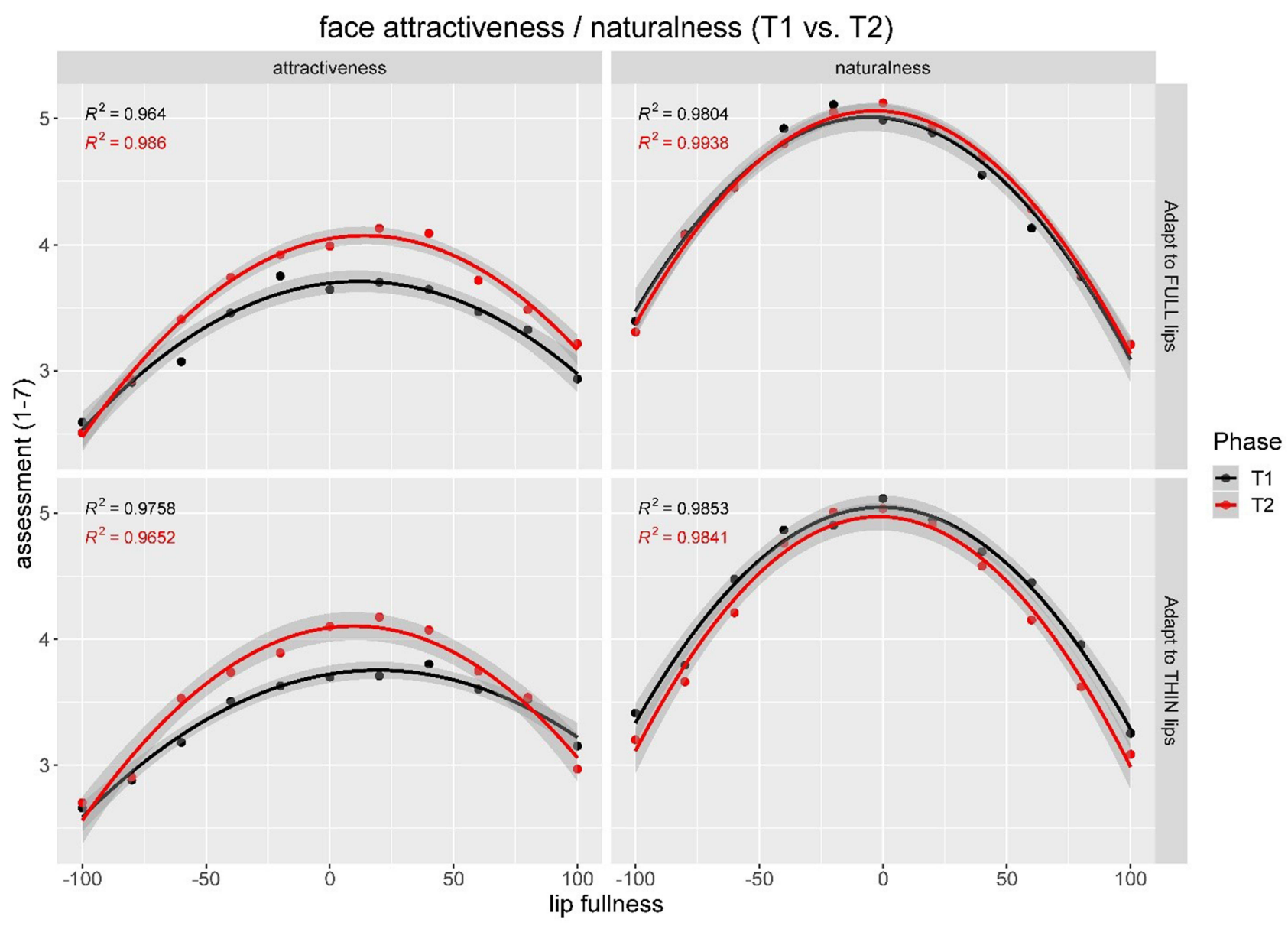

Figure 3 Mean data for face attractiveness (left) and face naturalness (right) for both adaptation conditions (top: Adapt_FullLips, bottom: Adapt_ThinLips), split by test phases (black: TI, red: T2). Data is modelled by second-degree polynomial functions-determination coefficient expressed as squared Pearson's $R$ is given for each curve fitting. Confidence intervals ( $\mathrm{Cl}-95 \%)$ are additionally given by shadowed confidence bands.

shift of maximal assessments of attractiveness for Adapt_FullLips vs Adapt_ThinLips: Whereas we detected a rightward shift for Adapt_FullLips, we detected a leftward shift for Adapt_ThinLips. For naturalness, in contrast, only a non-significant effect at a negligible effect size of Cohen's $d=0.100$ was obtained - numerically the direction of the effects, however, was the same as for attractiveness ratings.

\section{Discussion}

This study demonstrated that adaptation towards faces with extremely modified lips had a clear effect on the assessment of attractiveness, but not on the assessment of naturalness. Facial attractiveness is conjectured to be subject to adaptation as part of the visual diet's effect on the norm-based coding model of face perception. ${ }^{6}$ An individual's aesthetic preference was affected by viewing extreme image versions, but the participants' norms indicated by naturalness were not affected. We can only speculate about the reasons for this dissociative effect on both dependent variables. It is possible that the adaptive mechanisms were incapable of changing norms based on much larger sets of possible faces. Thus, assessments of facial attractiveness determine whether there is an averageness to the viewed facial image, whereas assessments of naturalness judge whether the facial image has a morphology that fits with that of the general population.

This multi-speciality study focused on how mere inspection of a face, with the aim of holistically evaluating facial features, can have profound effects on subsequent assessments in aesthetic practice. The viewing of facial images with extremely modified lips caused an almost immediate loss of aesthetic anchors with a shift in the calibration and norms of attractiveness in subjects.

In our increasingly digital society, tools such as Instagram facial and body filters allow individual features in facial images to be enlarged. These digital tools feed an 
Table I Optimal Lip Fullness That Corresponds to the Mean (M) Maximum Values of the Employed Second-Order Polynomial Models for Each Participant, Adaptation Condition and Test Phase, Calculated for Both Dependent Variables (Attractiveness and Naturalness), Separately

\begin{tabular}{|l|l|l|l|l|}
\hline & $\mathbf{M}_{\mathbf{T I}}(\boldsymbol{n})$ & $\mathbf{M}_{\mathbf{T 2}}(\boldsymbol{n})$ & $\mathbf{M}_{\text {diff }}$ (T2-TI) & Cohen's d \\
\hline Attractiveness & & & & \\
$\quad$ Adapt_FullLips & $7.63(23)$ & $14.04(24)$ & +6.40 & 0.282 (small), n.s. \\
Adapt_ThinLips & $17.84(2 \mathrm{I})$ & $2.99(18)$ & -14.84 & $-0.54 \mathrm{I}$ (medium) \\
\hline Naturalness & & & & \\
Adapt_FullLips & $-6.69(22)$ & $-3.69(23)$ & +3.00 & 0.256 (small), n.s. \\
Adapt_ThinLips & $-5.33(23)$ & $-5.43(23)$ & -0.10 & 0.005 (negligible), n.s. \\
\hline
\end{tabular}

Notes: The $n$ of the first two columns provides the number of individual datasets that could be modelled. The last two columns show the differences (and direction of difference) between mean data for T2 and T1, expressed by mean differences and the respective effect size (Cohen's $d$ ). Non-significant effects are indicated by usage of italics for the respective effect sizes. Qualification of effect sizes are added in parentheses following the suggestions of Cohen (1988) ${ }^{30}$.

unconscious zeal for larger and larger lips, and are part of a runaway selection process. As a result, norms around the appreciation of attractiveness are being continually recalibrated and powered by the enveloping tsunami of social media images and videos showing the real effects of arguably overtreated lips, and artificially doctored images.

Visual adaptation, the recalibration of ideals and norms of attractiveness, is accelerating in a positive feedback

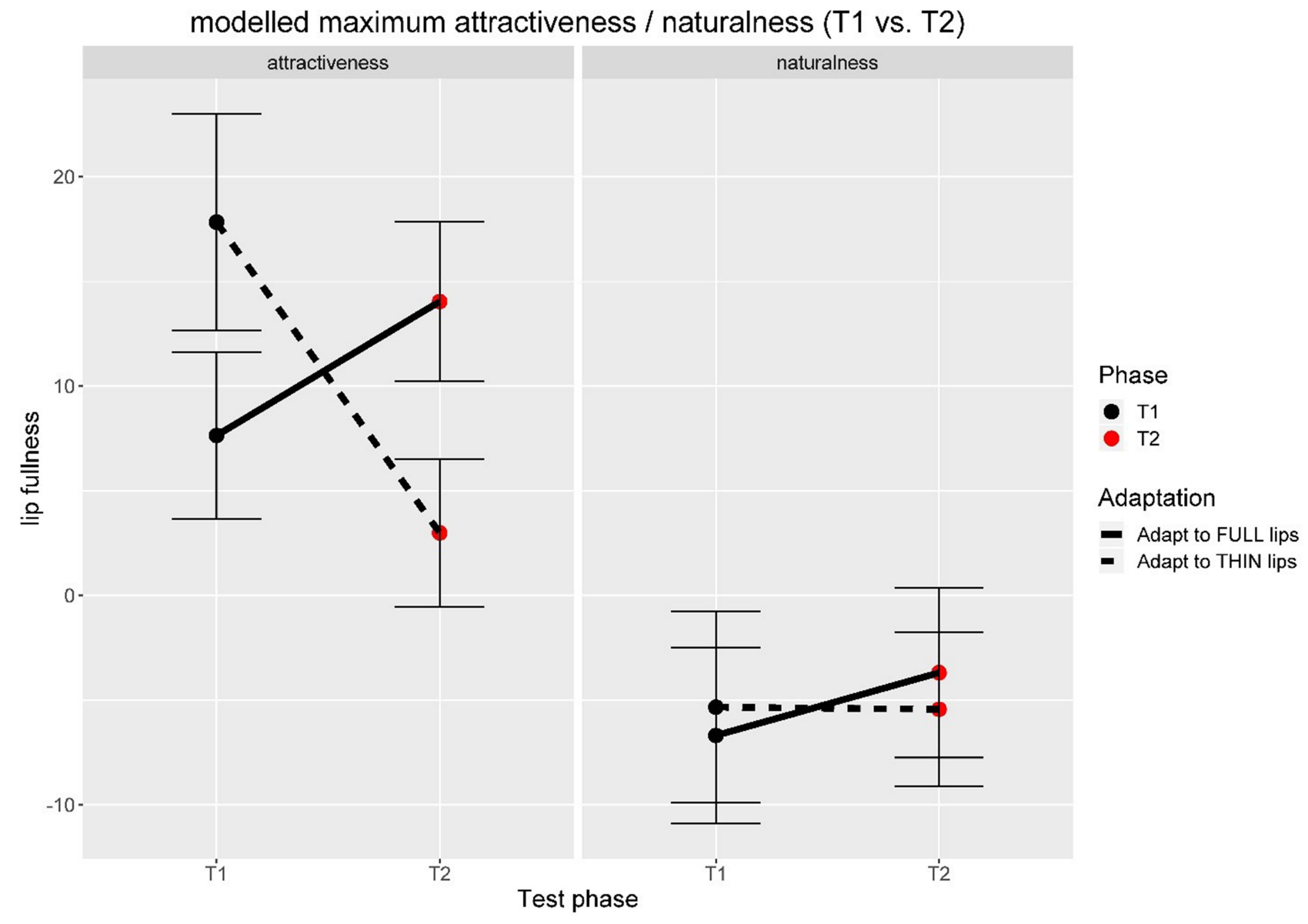

Figure 4 Mean values of individual optimal lip fullness for reaching a maximum for the respective dependent variables attractiveness (left) and naturalness (right). Solid lines show adaptation condition Adapt_FullLips and dashed lines the respective data for Adapt_ThinLips. Error bars indicate $\pm I$ standard error of the mean. 
loop - a runaway selective process not unlike the evolutionary processes powering the development of Peacock's tails and other traits throughout the biological world. ${ }^{29}$ Sub-groups of overtreated patients, and those persons, perhaps simultaneously overexposed to exaggerated, doctored images, develop and propagate aesthetic delusions now widespread and prominent in the 21 st century electronic culture.

This study shows that the adaptive process works in both directions, towards fuller lips and also towards thinner lips. This adaptive flexibility suggests that the aesthetic delusions are potentially reversible. An intelligent extrapolation of our results points to the possibility that patients with a bias towards finding full lips more attractive may be reoriented towards a "more normal" norm by the simple presentation of a set of thin-lip images. Future research efforts have to investigate the reversibility of adaptive effects with a view to helping both practitioners and patients manage their expectations.

The methodology of this study was designed so that the identity of the initial face pre-adaptations was different from the extreme Adapt_FullLips or Adapt_ThinLips faces. This helped to ensure that the phenomenon was truly transferable between faces, ie, that the shift in estimation of attractiveness would exist for all other faces seen and not just for that individual. This study did not evaluate how width lip affects attractiveness, and as the thickness of the lips is strongly influenced by width this is an area for future research.

In recent years, we have revealed facial adaptation effects lasting hours to days, after only very short exposures to stimuli with exaggerated features in lab-hosted ${ }^{12}$ as well as in more ecologically valid contexts. ${ }^{13}$ In modelling the extreme results of practitioners' occasional tendencies to overtreat, and the plethora of exaggerated images produced by rampant social media image manipulation, the study, in effect, asks an important question - "Do we know what beauty is?" These results suggest we do not. In our aesthetic clinics, and in the public's manipulations of images, do we risk losing our original identities to the casual drift of attractiveness calibration caused by adaptation?

\section{Conclusion}

The present paper provides significant insight into the potential for misjudgements and misperceptions by patients and medical practitioners in aesthetic assessments for surgical and nonsurgical interventions. Following exposure of participants to a series of modified, at times, even non-plausible or non- natural versions of faces, automatic adaptation resulted in almost instantaneous changes in the subjects' visual "beliefs" - their aesthetic appreciation. The infiltration of these induced changes into the adapted mind led to misjudgements biased towards frequently inspected facial images. The study emphasizes the importance of the visual diet, which when saturated with unrealistic media images and overtreated patients can be reflected in the mirrors of the mind and the creation of social groups with huge lips or over-large breasts - an induced automatic attractiveness calibration shift.

\section{Acknowledgments}

The authors wish to thank Merz Aesthetics for funding this collaborative cross-specialty research especially noting the assistance of Dr. T. Phillips, and her medical affairs team. The payment was only for conducting the study. CCC would like to thank Nathalie Schoemann, Yassin El Amri and Johannes Langer for their help in conducting this study and Vera M. Hesslinger for very fruitful discussion on the origin of the revealed adaptation effects. Photoshopped Liquify images are copyright of CCC.

\section{Funding}

The authors wish to thank Merz Aesthetics for funding this collaborative cross-specialty research. The payment was only for conducting the study.

\section{Disclosure}

The authors report no conflicts of interest in this work.

\section{References}

1. Aybay Y, Kara N. The impact of teen magazines on adolescent girls in North Cyprus. Soc Behav Pers. 2018;46(1):49-62. doi:10.2224/ sbp.6406

2. Holmstrom AJ. The effects of the media on body image: a meta-analysis. $J$ Broadcasting Electronic Media. 2004;48 (2):196-217. doi:10.1207/s15506878jobem4802_3

3. Tucci S, Peters J. Media influences on body satisfaction in female students. Psicothema. 2008;20(4):521-524.

4. Carbon CC. Cognitive mechanisms for explaining dynamics of aesthetic appreciation. I-Perception. 2011;2:708-719. doi:10.1068/i0463aap

5. Rhodes G, Jeffery L, Watson TL, Clifford CWG, Nakayama K. Fitting the mind to the world: face adaptation and attractiveness aftereffects. Psychol Sci. 2003;14(6):558-566. doi:10.1046/j.0956-7976.2003. psci_1465.x

6. Rhodes G, Jeffery L. Adaptive norm-based coding of facial identity. Vision Res. 2006;46(18):2977-2987. doi:10.1016/j.visres.2006.03.002

7. Chen HW, Russell R, Nakayama K, Livingstone M. Crossing the 'uncanny valley': adaptation to cartoon faces can influence perception of human faces. Perception. 2010;39(3):378-386. doi:10.1068/p6492

8. Principe CP, Langlois JH. Shifting the prototype: experience with faces influences affective and attractiveness preferences. Soc Cogn. 2012;30(1):109-120. doi:10.1521/soco.2012.30.1.109 
9. Carbon CC, Leder H. Face adaptation: changing stable representations of familiar faces within minutes? Adv Cogn Psychol. 2005;1 (1):1-7. doi:10.5709/acp-0038-8

10. Leopold DA, Rhodes G, Mueller KM, Jeffery L. The dynamics of visual adaptation to faces. Proc Biol Sci. 2005;272(1566):897-904.

11. Ditye T, Javadi AH, Carbon CC, Walsh V. Sleep facilitates long-term face adaptation. Proc Biol Sci. 2013;280(1769):20131698.

12. Carbon CC, Ditye T. Sustained effects of adaptation on the perception of familiar faces. J Exp Psychol Hum Percept Perform. 2011;37 (3):615-625. doi:10.1037/a0019949

13. Carbon CC, Ditye T. Face adaptation effects show strong and long-lasting transfer from lab to more ecological contexts. Front Psychol. 2012;3(3):1-6. doi:10.3389/fpsyg.2012.00003

14. Carbon CC, Strobach T, Langton S, Harsanyi G, Leder H, Kovacs G. Adaptation effects of highly familiar faces: immediate and long lasting. Mem Cognit. 2007;35(8):1966-1976. doi:10.3758/ BF03192929

15. Strobach T, Carbon CC. Face adaptation effects: reviewing the impact of adapting information, time, and transfer. Front Psychol. 2013;4:318. doi:10.3389/fpsyg.2013.00318

16. Carbon CC, Leder H. The Mona Lisa effect: is 'our' Lisa fame or fake? Perception. 2006;35(3):411-414. doi:10.1068/p5452

17. Germine L, Russell R, Bronstad PM, et al. Individual aesthetic preferences for faces are shaped mostly by environments, not genes. Curr Biol. 2015;25(20):2684-2689. doi:10.1016/j. cub.2015.08.048

18. Tiggemann M, Anderberg I. Social media is not real: the effect of 'Instagram vs reality' images on women's social comparison and body image. New Media Soc. 2019;22(12):2183-2199. doi:10.1177/ 1461444819888720

19. Seifert T. Anthropomorphic characteristics of centerfold models: trends towards slender, figures over time. Int J Eat Disord. 2005;37 (3):271-274. doi:10.1002/eat.20086

20. Norton KI, Olds TS, Olive S, Dank S. Ken and Barbie at life size. Sex Role. 1996;34(3-4):287-294. doi:10.1007/BF01544300
21. Yoon S, Kim YA. Cosmetic surgery and self-esteem in South Korea: a systematic review and meta-analysis. Aesthet Plast Surg. 2019;44 (1):229-238. doi:10.1007/s00266-019-01515-1

22. Rohrich RJ, Adams WP, Potter JK. A review of psychological outcomes and suicide in aesthetic breast augmentation. Plast Reconstruct Surg. 2007;119(1):401-408. doi:10.1097/01. prs.0000245342.06662.00

23. Webster MA, Kaping D, Mizokami Y, Duhamel P. Adaptation to natural facial categories. Nature. 2004;428(6982):557-561. doi:10.1038/nature02420

24. Zuckerman M. Item revisions in the sensation seeking scale form V (SSS-V). Pers Individ Dif. 1996;20(4):515. doi:10.1016/01918869(95)00195-6

25. Rammstedt B, John OP. Short version of the Big Five Inventory (BFI-K): development and validation of an economic inventory for assessment of the five factors of personality. Diagnostica. 2005;51 (4):195-206. doi:10.1026/0012-1924.51.4.195

26. Carbon CC, Leder H. The Repeated Evaluation Technique (RET): a method to capture dynamic effects of innovativeness and attractiveness. App Cogn Psychol. 2005;19(5):587-601. doi:10.1002/acp. 1098

27. Bates D, Mächler M, Bolker B, Walker S. Fitting linear mixed effects models using lme4. J Stat Software. 2015;67(1):48. doi:10.18637/jss. v067.i01

28. Carbon CC, Leder H. When feature information comes first! Early processing of inverted faces. Perception. 2005;34(9):1117-1134. doi:10.1068/p5192

29. Grammer K, Fink B, Møller AP, Thornhill R. Darwinian aesthetics: sexual selection and the biology of beauty. Biol Rev 2003;78:305-407. doi:10.1017/S1464793102006085

30. Cohen J. Statistical Power Analysis for the Behavioral Sciences. 2nd ed. Hillsdale, NJ: Lawrence Erlbaum Associates; 1988.

\section{Publish your work in this journal}

Clinical, Cosmetic and Investigational Dermatology is an international, peer-reviewed, open access, online journal that focuses on the latest clinical and experimental research in all aspects of skin disease and cosmetic interventions. This journal is indexed on CAS.
The manuscript management system is completely online and includes a very quick and fair peer-review system, which is all easy to use. Visit http://www.dovepress.com/testimonials.php to read real quotes from published authors. 